\title{
OntogÊNese do Sistema de Temporização - A Construção e as Reformas dos Ritmos BiolóGicos aO LONGO DA VIDA HuMANA
}

\author{
Luiz Menna-Barreto ${ }^{1}$ e Daniela Wey $^{\mathbf{2}}$ \\ Instituto de Ciências Biomédicas - USP
}

\begin{abstract}
Nesta revisão apresentamos fatos e comentários sobre a evolução dos sistemas de temporização ("relógios biológicos") na espécie humana. Na Introdução definimos alguns conceitos básicos da Cronobiologia que serão utilizados ao longo do artigo. Nas quatro seções subseqüentes, discutimos fatos marcantes que caracterizam a ritmicidade biológica em distintas etapas da ontogênese: bebês, adolescentes, adultos e idosos. Concluímos o artigo com um convite à reflexão sobre as perspectivas que se abrem com esse novo campo do conhecimento.
\end{abstract}

Descritores: Cronobiologia. Desenvolvimento humano. Ritmos biológicos. Ciclo vigília-sono.

\section{Introdução}

$\mathrm{O}$ objetivo deste artigo é oferecer um quadro sintético dos processos que marcam o desenvolvimento da ritmicidade biológica no homem. Boa parte dos exemplos utilizados derivam de pesquisas realizadas no âmbito do Grupo Multidisciplinar de Desenvolvimento e Ritmos Biológicos (GMDRB) do Instituto de Ciências Biomédicas da USP ao longo dos últimos vinte anos.

1 Grupo Multidisciplinar de Desenvolvimento e Ritmos Biológicos, Departamento de Fisiologia e Biofísica do Instituto de Ciências Biomédicas da Universidade de São Paulo. Endereço eletrônico: menna@usp.br

2 Endereço eletrônico: daniwey@icb.usp.br 
O cenário teórico dessas pesquisas é um ramo relativamente recente da Biologia, a Cronobiologia, que se ocupa do estudo da organização temporal da matéria viva (Marques \& Menna-Barreto, 2003).

Há dois aspectos que justificam o estudo do funcionamento dos seres vivos do ponto de vista temporal. Em primeiro lugar, o caráter dinâmico dos fenômenos vitais limita o alcance de observações nas quais a vida é cristalizada, como, por exemplo, em um tecido examinado em microscópio. Evidentemente, esse olhar tem o valor de um instantâneo, uma fotografia que congela o objeto. Em segundo lugar, a demonstração da existência de mecanismos internos produtores de tempo, os assim chamados "relógios biológicos", obriganos a reler muito do que sabíamos sobre as relações temporais dos organismos com seus ambientes. Hoje já não é possível entender as oscilações em nossos comportamentos como meras respostas a estímulos ambientais, na medida em que a responsividade dos organismos é sabidamente modulada pelos seus sistemas de temporização.

Um conceito fundamental da Cronobiologia é o de Organização Temporal Interna (Moore-Ede, Sulzman \& Fuller, 1982). Nessa organização, sintetiza-se a seqüência de eventos orgânicos que compõem um dia de um organismo. Em linhas gerais, ocorre o seguinte: algum tempo depois de iniciarmos nosso sono noturno, aumenta a produção do hormônio do crescimento, enquanto isso, já existe uma concentração elevada de outro hormônio, a melatonina, e as diversas fases do sono vão se sucedendo até um pouco antes de acordarmos, quando nossa temperatura corporal chega a seus valores mais baixos e um outro hormônio, o cortisol, tem sua concentração aumentada. Essa seqüência que culmina com o despertar tende a repetir-se diariamente e é modificada quando há uma alteração nas nossas relações temporais com o ambiente, como numa mudança de fuso horário, por exemplo. O mal-estar associado aos primeiros dias é a percepção que resulta da ruptura dessa Organização Temporal Interna e tende a dissipar-se à medida que nos adaptamos ao novo fuso horário e a seqüência descrita é resgatada.

Ao conceito de Organização Temporal Interna estamos propondo (DíezNoguera \& Menna-Barreto, em preparação) um desdobramento que consiste na

3 Preferimos a expressão "sistemas de temporização" a "relógios biológicos" por entender, com outros autores, que esta segunda expressão acaba induzindo os leigos a supor a existência de mecanismos de relojoaria nos organismos (Brandstaetter, 2004). 
Ontogênese do Sistema de Temporização - A Construção e as Reformas dos Ritmos...

identificação de uma Organização Temporal Externa, que se caracteriza pelas relações temporais entre eventos orgânicos (por exemplo, o sono ou a temperatura corporal) e eventos ambientais que têm reconhecido poder de sincronizar nossos ritmos biológicos, como é o caso do ciclo claro/escuro do dia e da noite, ou ainda o ciclo de marés para organismos que vivem na orla marítima.

Sabemos hoje que um organismo (humanos certamente incluídos) passa cotidianamente por processos de ajuste de seus ritmos, através da ação de ciclos ambientais que arrastam nossos sistemas de temporização. Em situações de isolamento temporal, nas quais são eliminados sinais que marcam a passagem do tempo, como é o caso de observações feitas em cavernas ou em laboratórios, os ritmos são preservados e tendem a exibir períodos maiores do que 24 horas $^{4}$. Os ritmos biológicos observados nessas condições de isolamento são conhecidos como "ritmos em livre-curso", ou seja, há ausência de sinais temporais no ambiente. Essa expressão em livre-curso aponta para uma origem endógena dos ritmos biológicos, o que acabou sendo demonstrado por meio da identificação de estruturas orgânicas (áreas do Sistema Nervoso Central) cuja integridade é essencial para a gênese da ritmicidade (Moore \& Lenn, 1972). Se a origem é reconhecidamente endógena, qual o papel dos sinais temporais do ambiente? Sabemos hoje que esses sinais atuam sobre os sistemas de temporização, sincronizando os ritmos através de processos conhecidos como arrastamento e mascaramento (Marques \& Menna-Barreto, 2003). Nem todos os sinais temporais atuam sobre os sistemas de temporização de todas as espécies e, ainda quando o mesmo ciclo ambiental sincroniza duas espécies, não estamos autorizados a supor que isso ocorra em ambas com a mesma força. Na espécie humana, o ciclo claro/escuro é reconhecidamente um dos mais importantes sinais ambientais capazes de "acertar nossos relógios", mas há também um papel importante desempenhado por estímulos sociais como horários de trabalho, lazer, refeições, enfim, interações sociais. $O$ fato de distintos ciclos ambientais exercerem influência sobre nossos sistemas de temporização promove uma relativa plasticidade que nos permite, por exemplo, alterarmos nossa rotina ou adaptarmo-nos a mudanças de fuso horário. No entanto, há limites para essa plasticidade, que impedem, por exemplo, a adaptação com-

4 O período dos ritmos em humanos nessas condições de isolamento temporal tende para 25h, no entanto, achados mais recentes apontam para valores de 24,2 ou 24,3 horas, de acordo com Czeisler (1999). 
pleta de nossos ritmos biológicos ao trabalho noturno (Moreno, Fischer, \& Rotenberg, 2002).

Os ritmos biológicos mudam ao longo da vida, alguns já estão presentes na vida fetal, outros só aparecem bem mais tarde (puberdade) e muitos alteram-se na velhice. Os ciclos ambientais capazes de promover a sincronização desses ritmos também não permanecem igualmente relevantes ao longo da vida. O conhecimento dos processos biológicos subjacentes pode nos ajudar a compreender os tempos que criamos e os tempos aos quais estamos submetidos ao longo da nossa existência. Nas seções a seguir, utilizaremos o ciclo vigília/sono como exemplo na maioria das mudanças ontogenéticas, tanto porque elas são bastante evidentes nesse ciclo, quanto porque também são as mais conhecidas e estudadas. Nossos exemplos privilegiarão os ritmos chamados circadianos, ou seja, aqueles que têm períodos entre 20 e 28 horas e que tendem a sincronizar-se com o dia social de 24 horas (Halberg, Carandente, Cornelissen, \& Katinas, 1977).

\section{Os primeiros ritmos as mães nunca esquecem.}

\subsection{Ambiente uterino do ponto de vista do bebê e de seus relógios}

O ambiente uterino é um local cercado por camadas de tecido epitelial e líquido amniótico, por esse motivo, poderíamos pensá-lo como um ambiente temporalmente constante e que o organismo que nele se desenvolve não apresenta ritmicidade. Mas, ao contrário disso, o feto tem ritmicidade já no útero, ambiente nada constante do ponto de vista temporal.

Por volta da $36^{\mathrm{a}}$ semana de gestação, o feto já desenvolveu um dos principais osciladores do sistema de temporização circadiano formado - os Núcleos Supra-Quiasmáticos (NSQs) - porém, as conexões nervosas responsáveis pelo funcionamento efetivo do sistema de temporização ainda estão imaturas (Reppert, 1992).

Antes do nascimento, todos os ritmos biológicos do feto estão sincronizados pelos ritmos maternos (Hoppenbrouwers et al., 1978). O ritmo atividade/repouso da mãe e as substâncias que chegam ao feto através do sangue ma- 
Ontogênese do Sistema de Temporização - A Construção e as Reformas dos Ritmos...

terno sinalizam um ambiente uterino rítmico. Essa ritmicidade uterina impede de dizer que o estabelecimento da ritmicidade biológica acontece apenas após o nascimento; antes disso, é possível observar que o feto apresenta momentos de maior atividade durante o dia e de menor atividade à noite. $\mathrm{O}$ processo de sincronização do feto pela mãe representa uma combinação de mecanismos de arrastamento e mascaramento sobre um sistema de temporização fetal em processo de maturação e ainda não submetido diretamente a ciclos ambientais, como o dia e a noite. A mãe acaba funcionando como um "pára-choque" temporal entre o ambiente e o feto (Marques \& Menna-Barreto, 2003).

\subsection{O nascimento é uma revolução temporal}

O nascimento marca uma mudança não só de ambientes mas de sinais temporais. Agora o bebê tem que se adaptar aos novos sinais. A influência materna se atenua (ou pelo menos muda bastante) após o nascimento, e o sistema de temporização do recém- nascido tem que se ajustar a uma nova realidade temporal, já não mais totalmente mediada pela mãe. Nessa época da vida, a informação temporal é proveniente principalmente da mãe ou da pessoa que cuida da criança.

Se observarmos essa transição da perspectiva temporal do bebê, veremos que ele tem que aprender a diferenciar entre dois momentos nos quais a principal pista temporal - a oscilação entre o dia e a noite - acontece concomitantemente com outros sinais não luminosos, como sons, movimentos e intervenções (trocar fralda, alimentar, dar banho...). Durante o dia, há muita luminosidade, movimento e intervenções; à noite, tudo isso tende a estar atenuado.

É dentro desse novo universo temporal que irá se reorganizar a ritmicidade do bebê. Os hábitos da família, sua rotina e seus horários também se modificam com a chegada do novo membro. Os horários de sono, principalmente da mãe, modificam-se de acordo com os despertares e os cochilos de seu bebê (Siegmund, Tittel \& Schiefenhövel, 1995). Parece razoável descrever essa época como de negociação de influências temporais entre mãe e criança, negociação tácita, mas não menos intensa e eventualmente até conflituosa, como veremos mais adiante. Essa reorganização temporal dos ritmos biológicos em 
relação ao novo ambiente é evidente quando observamos as mudanças que acontecem no padrão temporal do ciclo vigília/sono de bebês (Sadeh, 2001).

\subsection{A infância é um alinhamento dos ritmos}

O termo alinhamento está sendo usado aqui com duplo significado: de um lado, representa o processo de sincronização resultante da ação de ciclos ambientais sobre os sistemas de temporização; de outro, representa também o ajuste dos tempos da criança aos tempos sociais vigentes em seu ambiente. Cabe ressaltar que esses dois aspectos constituem dois lados de uma mesma moeda.

Após o nascimento, os ritmos biológicos em geral não apresentam periodicidade circadiana. Um recém-nascido dorme e acorda diversas vezes durante o dia, em ciclos que duram de 2 a 4 horas (Kleitman \& Engelmann, 1953; Parmelee, 1961). A presença desses componentes rápidos tem sido interpretada por alguns autores (Díez-Noguera, 1994) como evidência do processo de acoplamento entre osciladores mais rápidos, o que culminaria na expressão circadiana, pois, com o passar das semanas, o ciclo vigília/sono do bebê passa a se expressar com uma frequiência mais próxima das 24 horas. A instalação do padrão circadiano não é imediata e acontece no intervalo de uma semana a dois meses, caracterizada por importantes diferenças entre indivíduos (Bueno, Diambra \& Menna-Barreto, 2001; Menna-Barreto, Isola, Louzada, Mello, \& Benedito-Silva, 1996). Em diversos casos a ritmicidade circadiana já se faz presente mas ainda não está sincronizada aos ciclos ambientais, o que cria o quadro "meu filho troca o dia pela noite", que tende a ajustar-se sem necessidade de tratamento (Estivill \& Béjar, 2000; Sadeh, 2001).

Ao longo dos anos, os cochilos diurnos e os despertares noturnos da criança vão diminuindo, ela fica mais acordada durante o dia e com um sono noturno mais duradouro (Mello, Isola, Louzada \& Menna-Barreto, 1996). Como consequiência da redução dos cochilos diurnos, há uma redução nas horas totais de sono (Louzada, Orsoni, Mello, Benedito-Silva, \& Menna-Barreto, 1996).

Durante a infância, ocorrem diversas mudanças nas características do ciclo vigília/sono, contudo é impossível dizermos com precisão em que idade a criança deixará de cochilar ou de acordar no meio da noite, ou quantas horas 
Ontogênese do Sistema de Temporização - A Construção e as Reformas dos Ritmos...

de sono uma criança necessita em determinada idade (Klackenberg, 1982). Existem diferenças individuais importantes na evolução da expressão dos ritmos biológicos, até entre irmãos que moram na mesma casa (Healy, 1972). Há, entretanto, indicações de generalidades como, por exemplo, evidências de que meninas têm uma duração de sono maior do que meninos (Menna-Barreto, Soussignan, Montagner \& Koch, 1989).

Mas essas diferenças são atenuadas quando a criança começa a freqüentar a escola; os horários das atividades tornam os hábitos de sono muito semelhantes entre crianças de uma mesma faixa etária (Louzada, et al. 1996; Sadeh, Raviv, \& Gruber, 2000). Nessas crianças, já é possível perceber uma diferença nos horários de sono entre dias letivos e não letivos, isto é, há maior duração de sono nos dias não letivos, quando não existem horários impostos para acordar (Andrade \& Louzada, 2002; Gulliford, Price, Rona, \& Chinn, 1990; Koch, Soussignan \& Montagner, 1984).

\subsection{Cochilar é preciso?}

Dentre as mudanças verificadas nos hábitos de sono, observa-se que a redução ou o desaparecimento dos episódios de cochilo muitas vezes acontece como consequiência dos horários escolares e não de maneira natural. No ambiente escolar existem diversos estímulos sociais que acabam mascarando a real necessidade de cochilar para algumas crianças (Louzada \& Menna-Barreto, 2004). Com o passar dos anos, a necessidade de cochilar realmente diminui, mas, como foi mencionado anteriormente, existem diferenças individuais, variando a idade em que isso ocorre (Koch et al., 1984; Weissbluth, 1995).

Num estudo realizado por nós em escola de período integral em São Paulo, o Centro Educacional Brandão, observamos que, aos seis anos de idade, aproximadamente, $63 \%$ das crianças ainda cochilavam mais que 3 vezes por semana. Quando estas mesmas crianças entraram na primeira série, a necessidade de cochilar diminuiu para 37\% das crianças (Wey, 2002). Nessa escola, no ano de 2000, foi introduzida a possibilidade de um cochilo diário de cerca de uma hora no início da tarde para as crianças de 3 a 6 anos. Acompanhamos essa mudança e pudemos constatar que não houve prejuízo do sono noturno e, nalguns casos, testemunhamos depoimentos de professores constatando melho- 
ra no desempenho das crianças. Essas observações podem contribuir para um planejamento mais adequado de horários na pré-escola. $\mathrm{O}$ fato de uma criança poder ou não cochilar dentro de uma escola ou numa creche, quando tem necessidade, influencia diretamente o seu nível de atenção e traz conseqüências para o seu desempenho e a aprendizagem (Dahl, 1996).

\section{A adolescência atravessa uma crise temporal}

\subsection{Hormônios e ritmos}

As oscilações das concentrações de hormônios no nosso sangue podem ser vistas sob dois aspectos: aquele dos efeitos específicos dos hormônios (por exemplo, na indução de distribuição de pêlos e desenvolvimento de mamas) e aquele do sinal temporal representado pelos ciclos de produção hormonal. Um exemplo típico desse segundo aspecto é o do sinal temporal do hormônio melatonina, produzido pela glândula pineal do nosso cérebro. Esse hormônio, conhecido como "hormônio da noite interior", tem um padrão noturno de presença na corrente sangüínea e muitos pesquisadores interpretam esse fato como sinal interno da noite exterior, daí a denominação. Outros hormônios cujo padrão circadiano é bem conhecido são o hormônio do crescimento e o cortisol (Aschoff, 1979). No final da década de 1980, apareceu um debate até então ausente na literatura científica, mas que seguramente já envolvia pais e professores: a alta incidência de sonolência diurna em adolescentes (Andrade et al., 1993). Levantamentos dessa queixa sugeriam que se tratava de um fenômeno pouco importante na infância e que se manifestava amplamente nos adolescentes. O resultado dessa constatação originou uma série de pesquisas cujos resultados apontam para uma relação forte entre a queixa de sonolência e o desenvolvimento puberal; tratar-se-ia de resultado da ação direta ou indireta dos hormônios sexuais (Carskadon, Vieira \& Acebo, 1993). A explicação mais difundida para esse fenômeno refere-se a um "atraso da fase do sono", remetendo, portanto, para uma mudança na atuação dos sistemas de temporização, abrindo caminho para propostas de intervenção farmacológica (Gilbert, Van Den Heuvel, \& Dawson, 1999) e investigação sobre a base molecular do fenômeno (Ebisawa et al., 2001). 
Ontogênese do Sistema de Temporização - A Construção e as Reformas dos Ritmos...

\subsection{O cenário social atrasa relógios}

Complementando essa visão hormonal do atraso de fase do sono em adolescentes, há que se considerar a atuação de sincronizadores sociais, representados pelas rotinas familiares (e o eventual rompimento de regras pelos jovens) e horários de escola e/ou trabalho. Há inúmeros atrativos, na rua ou no ambiente doméstico (TV, internet), que tendem a fazer com que os jovens "empurrem" o horário de dormir para mais tarde, e o horário das aulas ou do trabalho exige que eles levantem cedo - está criada a armadilha temporal que comprime o sono e que produz o que vem sendo conhecido como "padrão sanfona" do ciclo vigília/sono: sono insuficiente durante a semana, sono prolongado no final de semana (Valdez, Ramirez, \& Garcia, 1996). Resultados de estudos do nosso laboratório (Louzada \& Menna-Barreto, 2003), nos quais comparamos o ciclo vigília/sono de adolescentes que vivem em ambientes rurais e urbanos, fazemnos considerar esse fenômeno do atraso de fase como algo marcadamente urbano - não encontramos evidência desse atraso nos jovens que vivem no campo.
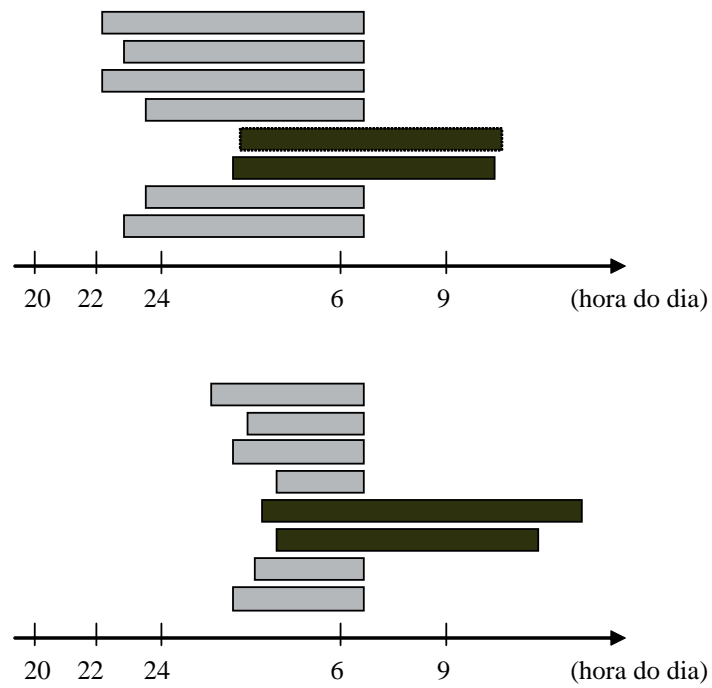

Figura 1. Representação hipotética dos padrões de duração do sono noturno de dois adolescentes ao longo de uma semana. Os finais de semana (sono das sextas para os sábados e dos sábados para os domingos) estão indicados pelas barras mais escuras. Observe-se o atraso de fase nos dois gráficos e o "padrão sanfona" no gráfico inferior, com evidências de restrição de sono nos dias de semana e extensão no final de semana, padrão que não ocorre no exemplo do gráfico superior. 


\subsection{Os conflitos com horários escolares}

Muitas escolas funcionam em turnos, especialmente escolas públicas nas quais é freqüente a mudança do turno da tarde para o da manhã quando as crianças têm em torno de 10-11 anos, ou seja, às vésperas ou já no início da puberdade. Ora, se os hormônios que agem sobre os sistemas de temporização e o ambiente social empurram os jovens para horários mais tardios e a escola exige que acordem mais cedo, eis a armadilha temporal mecionada. Resulta dela uma privação crônica de sono, com as indesejáveis conseqüências sobre o humor, a concentração, a memória e a aprendizagem (Dahl \& Lewin, 2002). Tivemos experiências muito interessantes em escola na qual pesquisamos essa armadilha: na Escola de Aplicação da FEUSP, mostramos esse efeito na transição tarde-manhã da $4^{\mathrm{a}}$ para a $5^{\mathrm{a}}$ série e, mais tarde, da $5^{\mathrm{a}}$ para a $6^{\mathrm{a}}$ série. Essa escola iniciou um processo de reforma temporal em 1999 (inversão completa dos turnos) que infelizmente foi interrompido em 2001, apesar dos efeitos de privação de sono claramente evidenciados (Mello, Louzada \& Menna-Barreto, 2001).

\section{Adultos estilhaçados no tempo}

\subsection{Horários de trabalho}

Há alguns anos, fazemos uma pesquisa informal perguntando se as pessoas consideram o sono como prazer ou obrigação, o que em geral produz amplos discursos sobre "respeito à saúde" no que tange ao prazer e "pura perda de tempo" no que diz respeito à obrigação. Raramente obtivemos conciliações. A quantidade de pessoas que dorme por obrigação (estimamos que seja a metade) revela tanto a ampla difusão de preconceitos contra os dorminhocos quanto o desconhecimento a respeito da necessidade de sono. Esses preconceitos, ligados à ignorância sobre o tema, criam o cenário favorável para que se montem armadilhas temporais com crescente intensidade, especialmente nos ambientes urbanos (Coren, 1996). A mais freqüente está nos horários irregulares de trabalho, tipicamente no esquema de turnos alternantes, nos quais se trabalha uma semana pela manhã, na semana seguinte à tarde e na terceira semana à noite. A suposição é de que somos capazes de nos adaptar a essas mudanças 
Ontogênese do Sistema de Temporização - A Construção e as Reformas dos Ritmos...

sem maiores custos. Sabemos hoje que esse é um grande equívoco e há uma literatura considerável a respeito dos problemas de saúde associados a esses esquemas irregulares de trabalho (Fischer, Moreno, \& Rotenberg, 2003). Do ponto de vista da Cronobiologia, o que chama a atenção nesses estudos é o efeito de atenuação ou mesmo de abolição da ritmicidade biológica que caracteriza o indivíduo saudável. Trabalhadores em turnos ou noturnos são freqüentemente indivíduos desorganizados temporalmente, nos quais a sequiência de eventos que caracteriza a Organização Temporal Interna e Externa está rompida.

Uma questão que tem surgido com bastante insistência nos últimos anos é a dos limites dentro dos quais mudanças de horários seriam toleráveis. Sabe-se que, além de características individuais distintas (matutinidade/vespertinidade e necessidade de sono) que conferem maior ou menor tolerância, a idade está negativamente correlacionada com essa tolerância (Härmä, 1996).

\subsection{Mudanças de fuso}

Viagens aéreas transmeridianas acarretam mudanças bruscas de fuso horário e consequiente desorganização temporal de intensidade proporcional à diferença entre os fusos de origem e de destino do passageiro. Esse efeito, mais conhecido pela expressão inglesa jet lag, tem representado um desafio para pesquisadores que buscam a cura ou, pelo menos, a atenuação do malestar que caracteriza o estado de dessincronização. Exposição à luz, ingestão de melatonina e exercícios físicos têm sido propostos como medidas para acelerar o processo de ressincronização (Marques \& Menna-Barreto, 2003). Para quem viaja e permanece no local de destino, o problema é passageiro, mas o mesmo não ocorre com quem viaja freqüentemente, como é o caso de tripulações de avião. Para elas, a situação equivale àquela do trabalhador em turnos ou noturno: quando se está prestes a sincronizar, muda-se de horário, o que cronifica a dessincronização. Chama a atenção o desacoplamento de ritmos que tende a ocorrer nessas situações, nas quais o ciclo vigília/sono tende a se ajustar bem mais rapidamente do que outros ritmos, como o da temperatura corporal (Ashoff, Gerecke, \& Wever, 1967). Uma solução mais eficiente para minorar o jet lag virá do conhecimento mais preciso da potência dos sinais temporais ambientais e orgânicos envolvidos nesse processo. 


\subsection{Doenças temporais - agudas e crônicas}

A depressão unipolar tem sido associada a distúrbios dos sistemas de temporização, sendo que alguns pesquisadores sugerem que esses distúrbios podem se constituir em componentes da gênese da depressão (Wehr \& WirzJustice, 1982). Causa ou efeito, a desorganização temporal se faz presente na depressão e em outras patologias, o que nos permite propor que intervenções cujo objetivo seja a recomposição da Organização Temporal Externa e Interna devam fazer parte do arsenal terapêutico. Há diversas evidências segundo as quais um organismo sincronizado reage melhor a condições adversas, como processos infecciosos, por exemplo, (Katz, Marpegan, \& Bekinschtein, 2002).

A desorganização temporal pode ser aguda, como na mudança brusca de fuso horário, ou crônica, como acontece com trabalhadores em horários irregulares. Nos casos crônicos, a intervenção se faz necessária tanto no sentido mais efetivo de eliminar as causas (redesenhando horários de trabalho, por exemplo), quanto no sentido de atenuar os efeitos adversos como fadiga, sonolência, insônia, etc.

Hoje identificamos patologias do próprio sistema de temporização: as chamadas síndromes do ciclo vigília/sono diferente de 24 horas e da fase atrasada ou adiantada do sono. Na primeira síndrome, trata-se de indivíduos que espontaneamente apresentam ciclos em geral maiores do que $24 \mathrm{~h}$, e que tendem a dormir cada dia mais tarde, o que gera problemas sobretudo na esfera das relações sociais. A outra síndrome é bastante discutível, pois, na verdade, refere-se a tendências extremas à matunidade (fase adiantada) ou à vespertinidade (fase atrasada). Uma reflexão provocativa sobre essas características individuais foi proposta por Alan Fogel (1999) em um ensaio no qual compara sua história pessoal de matutino com a história de um colega vespertino e de como as temporalidades distintas marcaram essas histórias. 
Ontogênese do Sistema de Temporização - A Construção e as Reformas dos Ritmos...

\section{Idosos voltam no tempo?}

\subsection{Fragmentação e avanço do ciclo vigília/sono}

Na senescência, o ciclo vigília/sono apresenta características distintas dos padrões observados no adulto. Ocorrem modificações no sistema de temporização e também na relação que existe entre o organismo e as pistas temporais ambientais (Pandi-Perumal et al., 2002). Durante o desenvolvimento humano, não apenas o organismo se modifica, mas também o modo como nos relacionamos temporalmente com o mundo.

De maneira geral, observa-se que o horário de início do sono dos idosos passa a acontecer mais cedo e esse avanço de fase é observado também para outros ritmos biológicos, como o ciclo de temperatura corporal, de secreção de hormônios, o ciclo de atividade/repouso, etc. (Brock, 1991; Copinschi \& Van Cauter, 1995; Monk, Buysse, Reynolds Iii, Kupfer, \& Houck, 1995; Renfrew, Pettigrew, \& Rapoport, 1987). O fato de o idoso acordar mais cedo pode estar relacionado ao aumento da quantidade de sono sincronizado e superficial na parte final do sono e, com isso, o indivíduo fica mais suscetível aos estímulos ambientais (Dijk, Duffy, \& Czeisler, 2000).

Uma outra mudança observada está na continuidade do sono noturno. No idoso, o ciclo vigília/sono se apresenta mais fragmentado, tal como acontecia no início da vida, com uma incidência maior de despertares noturnos e cochilos diurnos (Carskadon, Vieira, \& Acebo, 1982). Essa característica parece decorrer de uma redução na amplitude dos ritmos biológicos observada, por exemplo, no ciclo de temperatura corporal. Como consequiência da fragmentação do ciclo vigília/sono nos idosos, observa-se uma perda na relação de fase entre os ritmos biológicos, conhecida como desorganização temporal interna. Além disso, observa-se que o idoso apresenta uma dificuldade maior do que indivíduos jovens para ser sincronizado pelos mesmos estímulos sociais, o que promove a desorganização temporal externa (Samis Jr., 1968; Turek et al., 1995; Van Gool \& Mirmiran, 1986).

Num organismo, em qualquer etapa de desenvolvimento, existem ritmos com expressão mais robusta e menos suscetíveis a modulações, como é o caso da temperatura central, e há outros que modificam facilmente sua 
expressão pela ação de mascaradores externos ou internos, como é o caso do ciclo vigília/sono. No idoso, o ciclo de temperatura central tem uma amplitude reduzida, mas se expressa com uma freqüência circadiana característica. Já o ciclo vigília/sono está fragmentado, o que provoca uma desestabilização na relação de fase entre esses dois ritmos. O idoso apresenta episódios de sono em diversos momentos ao longo da variação do ciclo de temperatura corporal, quando anteriormente havia uma coincidência entre a ocorrência de sono e os valores mais baixos da temperatura. A desorganização temporal interna entre os ritmos biológicos do idoso tem como conseqüência uma redução na qualidade de sono, relatada por esses indivíduos (Ceolim, Campedelli, \& MennaBarreto, 1996). Alguns pesquisadores argumentam que a qualidade de sono influencia fortemente a qualidade de vida do indivíduo, pois uma boa noite de sono tem reflexos sobre as atividades do dia seguinte; para indivíduos idosos, uma melhor qualidade de vida pode resultar num aumento da expetativa de vida (Stampi, 1992).

Uma outra consequiência das mudanças observadas na característica dos ritmos biológicos do indivíduo idoso é uma alteração nas suas relações sociais. Os horários de sono são diferentes dos indivíduos mais jovens e, além disso, eles freqüentemente se queixam da dificuldade em se manter acordados durante o dia e de iniciar e manter o sono noturno (Foley et al., 1995).

Na tentativa de entender melhor as modificações no processo de ontogênese humana, os pesquisadores formularam três hipóteses sobre os mecanismos subjacentes ao sistema de temporização. A primeira está centrada no papel do ambiente cíclico agindo na sincronização desses indivíduos: no idoso, a mudança em seus ritmos biológicos pode decorrer de uma quantidade insuficiente de pistas temporais ambientais à qual o indivíduo está submetido (Monk, 1989; Rosenberg, 1982). A segunda hipótese remete ao funcionamento do sistema sensorial e das vias envolvidas na percepção e na decodificação do sinal temporal (Rosenberg, 1982). No processo de envelhecimento, ocorreria a deterioração do organismo e o prejuízo de diversas funções necessárias à sobrevivência, uma dessas mudanças está na acuidade de sistemas sensoriais, como a audição e a visão, por exemplo. A terceira hipótese busca explicar as alterações no sistema de temporização do idoso considerando um prejuízo no funcionamento do sistema de temporização circadiana (Moore-Ede et al., 
Ontogênese do Sistema de Temporização - A Construção e as Reformas dos Ritmos...

1982, Turek et al., 1995; Webb, 1987): com o envelhecimento, ocorreriam modificações anatômicas e funcionais desse sistema, que promoveriam alterações dos ritmos biológicos; a fragmentação do sono seria uma das evidências dessa mudança nos ritmos. A importância da integridade de um oscilador endógeno na expressão dos ritmos circadianos foi evidenciada num experimento no qual o transplante de núcleos supraquiasmáticos fetais foi capaz de restaurar o padrão circadiano de oscilação do ritmo de atividade e repouso em camundongos idosos (Hurd \& Ralph, 1998). As características dos ritmos biológicos de idosos - fragmentação e avanço de fase - podem ser explicadas por qualquer uma das hipóteses anteriores ou pela combinação delas.

\subsection{Fatalidade ou artefato?}

Para a maioria dos idosos e indivíduos aposentados, outras pistas temporais, diferentes dos horários de trabalho, passam a ter importância no processo de arrastamento dos ritmos circadianos. Algumas pesquisas indicam que a redução das atividades sociais, que acontecem, por exemplo, quando o indivíduo se aposenta, ou a ausência de uma rotina diária regular poderiam promover a desorganização temporal de alguns ritmos biológicos (Ceolim et al., 1999). Como consequiência da desorganização temporal do ciclo vigília/ sono, pode haver uma redução na qualidade de sono até o desenvolvimento de estados depressivos.

As mudanças no sistema de temporização observadas no final da ontogênese podem se apresentar de maneira atenuada para alguns idosos, sobretudo aqueles que têm uma rotina diária e participam de atividades sociais regularmente. Num estudo realizado por Ceolim (2000), foi possível verificar que a fragmentação do sono noturno estava quase ausente em idosos extremamente saudáveis, que praticavam esportes regularmente e se expunham à luz solar pela manhã. As alterações nos padrões do sono do idoso parecem ser decorrentes mais de seus hábitos e condições de vida do que do envelhecimento biológico. 
Luiz Menna-Barreto e Daniela Wey

\title{
6. A reflexão necessária - para qual tempo nos encaminhamos?
}

Uma projeção linear das descobertas até este momento aponta para uma sociedade que controla tecnicamente o tempo biológico, seja no processo de aceleração da sincronização no início e ao longo da ontogênese, seja no processo de retardamento da desorganização na etapa final da ontogênese. A expectativa média da vida humana vem sendo alargada nas últimas décadas em função da qualidade/quantidade de alimentos e recursos da saúde. A novidade é que agora contemplamos a possibilidade de intervir na intimidade dos processos que determinam a duração da vida. Em invertebrados já existem evidências de que esse controle é possível (Kinney-Forshee, Kinney, Steger, \& Bartke, 2004; Seong, Ogashiwa, Matsuo, Fuyama, \& Aigaki, 2001). O que nos parece tarefa atual relevante é associarmos o conhecimento dos mecanismos ao re-conhecimento das situações que criamos e às quais nos submetemos ao longo da vida.

Menna-Barreto, L., \& Wey, D. (2007). Ontogeny of the timing system - building and reforms of biological rhythms along human life. Psicologia USP, 18(2), 133-153.

\begin{abstract}
In this review we present facts and comments on the evolution of the timing systems ("biological clocks") in humans. In the Introduction we define basic concepts of Chronobiology which will be used along the paper. In the four following sections we discuss landmarks which characterize biological rhythmicity in distinct moments of ontogeny: infants, adolescents, adults and elderly. We close the review with an invitation to consider the perspectives now open in this new area of knowledge.
\end{abstract}

Index terms: Chronobiology. Human development. Biological rhythms. Sleep wake cycle. 
Ontogênese do Sistema de Temporização - A Construção e as Reformas dos Ritmos...

Menna-Barreto, L., \& Wey, D. (2007). L'ontogenèse du système de temporisation - sa constrution et les changements de rythmes biologiques au long de la vie humaine. Psicologia USP, 18(2), 133153.

Résumé: Cette révision présente certains faits et des commentaires sur l' ontogenèse des systèmes de temporisation («les horloges biologiques») chez l'homme. D’abord, nous présentons quelques définitions fondamentales de la Chronobiologie, ce qui nous semble nécessaire à la compréhension du texte suivant. Les quatre sections organisées à la suite abordent des faits remarquables qui caractérisent la rhythmicité biologique au cours de l'ontogenèse chez les bébés, les adolescents, les adultes et les anciens. À la conlusion nous proposons une réflexion sur les perspectives qui s’ouvrent par ce nouveau champ de connaissance.

Most-clés: Chronobiologie. Développement humain. Rythmes biologiques. Dormir le cycle de suite.

\section{Referências}

Andrade, M. M. M., Benedito-Silva, A. A., Domenice, S., Arhold, I. J. P., \& Menna-Barreto, L. (1993). Sleep characteristics of adolescents: A longitudinal study. The Journal of Adolescent Health, 14, 401-406.

Andrade, M. M. M., \& Louzada, F. (2002). Ritmos biológicos en ambientes escolares. In D. Golombek (Org.), Cronobiología humana: ritmos y relojes biológicos en la salud y en la enfermedad. Buenos Aires: Universidad Nacional de Quilmes Ediciones.

Aschoff, J. (1979). Circadian rhythms: General features and endocrinological aspects. In D. T. Krieger (Ed.), Endocrine rhythms (pp. 1-61). New York: Raven.

Aschoff, J., Gerecke, U., \& Wever, R. (1967). Desynchronization of human circadian rhythms. Japan Journal of Physiology, 17, 450-457.

Brandstaetter, R. (2004). Circadian lessons from peripheral clocks: Is the time of the mammalian pacemaker up? Proceedings of the National Academy of Science, 101(16), 5699-5700.

Brock, M. A. (1991). Chronobiology of aging. Journal of the American Geriatrics Society, 39, 79-91.

Bueno, C., Diambra, L., \& Menna-Barreto, L. (2001). Sleep/wake and temperature rhythms in preterm babies maintained in a neonatal care unit. Sleep Research Online, 4(3), 77-82. 


\section{Luiz Menna-Barreto e Daniela Wey}

Carskadon, M. A., Vieira, C., \& Acebo, C. (1993). Association between puberty and delayed phase preference. Sleep, 16(3), 258-262.

Carskadon, M. A., Brown, E. D., \& Dement, W. C. (1982). Sleep fragmentation in the elderly: Relationship to daytime sleep tendency. Neurobiology of Aging, 3, 321-327.

Ceolim, M. F. (2000). Sleep/wake cycle and physical activity in healthy elderly people. Sleep Research Online, 3(3), 87-95.

Ceolim, M. F., Campedelli, M. C., \& Menna-Barreto, L. (1996). Circadian amplitude and quality of sleep in a group of elderly subjects. Biological Rhythms Research, 27(3), 398408.

Copinschi, G., \& Van Cauter, E. (1995). Effects of ageing on modulation of hormonal secretions by sleep and circadian rhythmicity. Hormone Research, 43, 20-24.

Coren, S. (1996). Ladrões de sono. (R. G. de Souza, trad.). São Paulo: Cultura Editores Associados.

Czeisler C. A., Duffy J. F., Shanahan, T. L., Brown, E. N., Mitchell, J. F., Rimmer, D. W., et al. (1999). Stability, precision, and near-24-hour period of the human circadian pacemaker. Science, 284(5423), 2177-2181.

Dahl, R. E. (1996). The impact of inadequate sleep on children's daytime cognitive function. Seminars in Pediatric Neurology, 3(1), 44-50.

Dahl, R. E., \&. Lewin, D. S. (2002). Pathways to adolescent health: Sleepn regulation and behavior. Journal of Adolescent Health, 31, 175-184.

Dijk, D.-J., Duffy, J. F., \& Czeisler, C. A. (2002). Contribution of circadian physiology and sleep homeostasis to age-related changes in human sleep. Chronobiology International, 17(3), 285-312.

Díez-Noguera, A. (1994). A functional model of the circadian system based on the degree of inter-communication in a complex system. The American Journal of Physiology, 267, R1118-1135.

Ebisawa, T., Uchiyama, M., Kajimura, N., Mishima, K., Kamei, Y., Katoh, M., et al. (2001). Association of structural polymorphisms in the human period 3 gene with delay sleep phase syndrome. EMBO Rep, 2(4), 342-346.

Estivill, E., \& Béjar, S. (2000). Nana, nenê: como resolver os problemas da insônia infantil (M. Stahel, trad.). São Paulo: Martins Fontes.

Fischer, F. M., Moreno, C. R. C., \& Rotenberg, L. (2003). Trabalho em turnos e noturno na sociedade 24 horas. São Paulo: Atheneu.

Fogel, A. (1999). Systems, cycles, and developmental pathways. Human Development, 42, 213-215. 
Ontogênese do Sistema de Temporização - A Construção e as Reformas dos Ritmos...

Foley, D. J., Monjan, A. A., Brown, S. L., Simonsick, E. M., Wallace, R. B., \& Blazer, D. G. (1995). Sleep complaints among elderly persons: an epidemiological study of three communities. Sleep, 18, 425-432.

Gilbert, S. S., Van Den Heuvel, C. J., \& Dawson, D. (1999). Daytime melatonin and temazepan in young adult humans: Equivalent effects of sleep latency and body temperature. Journal of Physiology, 514, 905-914.

Gulliford, M. C., Price, C. E., Rona, R. J., \& Chinn, S. (1990). Sleep habits and height at ages 5 to 11. Archives of Disease in Childhood, 65, 119-22.

Halberg, F., Carandente, F., Cornelissen, G., \& Katinas, G. S. (1977). Glossary of chronobiology. Chronobiologia, 4(Suppl. 1 1), 67-68

Härmä, M. (1996). Ageing, physical fitness and shiftwork tolerance. Applied Ergonomics, $27(1), 25-29$.

Healy, A. (1972). The sleep patterns of preschool children. Clinical Pediatrics, 11(3), 174-177.

Hoppenbrouwers, T., Ugatechea, J. C., Combs, D., Hodgman, J. E., Harper, R. M., \& Sterman, M. B. (1978). Studies of maternal-fetal interaction during the last trimester of pregnancy: Ontogenesis of the basic-rest activity cycle. Experimental Neurology, 61, 136-153.

Hurd, M. W., \& Ralph, M. R. (1998). The significance of circadian organization for longevity in the golden hamster. Journal of Biological Rhythms, 13, 430-436.

Katz, M., Marpegan, L., \& Bekinschtein, T. (2002). Ritmos diarios y circadianos en el sistema inmunologico. In D. Golombek (Org.), Cronobiología humana: ritmos y relojes biológicos en la salud y en la enfermedad. Buenos Aires: Universidad Nacional de Quilmes Ediciones.

Kinney-Forshee, B. A., Kinney, N. E., Steger, R. W., \& Bartke, A. (2004). Could a deficiency in growth hormone signaling be beneficial to the aging brain? Physiology and Behavior, 80(5), 589-594.

Klackenberg, G. (1982). Sleep behaviour studied longitudinally. Acta Paediatrica Scandinavica, 71, 501-506.

Kleitman, D. D., \& Engelmann, T. G. (1953). Sleep characteristics of infant. Journal Applied Physiology, 6, 269-282.

Koch, P., Soussignan, R., \& Montagner, H. (1984). New data on the wake-sleep rhythm of children aged from $2 \frac{1}{2}$ to $4 \frac{1}{2}$ years. Acta Paediatrica Scandinavica, 73, 667-673.

Louzada, F., \& Menna-Barreto, L. (2003). Sleep-wake cycle expression in adolescence: Influences of social context. Biological Rhythm Research, 34(2), 129-136.

Louzada, F., \& Menna-Barreto, L. (2004). Relógios biológicos e aprendizagem. São Paulo: Editora do Instituto Esplan. 


\section{Luiz Menna-Barreto e Daniela Wey}

Louzada, F. M., Orsoni, A., Mello, L., Benedito-Silva, A. A., \& Menna-Barreto, L. (1996). A longitudinal study of the sleep/wake cycle in children living on the same school schedules. Biological Rhythm Research, 27(3), 390-397.

Marques, N., \& Menna-Barreto, L. (Eds.). (2003). Cronobiologia: princípios e aplicações (3a ed.). São Paulo: EDUSP.

Mello, L., Isola, A., Louzada, F. M., \& Menna-Barreto, L. (1996). A four-year follow-up study of the sleep-wake cycle of an infant. Biological Rhythm Research, 27(3), 291-298.

Mello, L., Louzada, F. M., \& Menna-Barreto, L. (2001). Effects of school schedules transition on sleep/wake cycle of Brazilian adolescents. Sleep \& Hypnosis, 3(3), 106-111.

Menna-Barreto, L., Isola, A., Louzada, F. M., Mello, L., \& Benedito-Silva, A. A. (1996). Becoming circadian - a one-year study of the development of the sleep-wake cycle in children. Brazilian Journal Medical Biological Research, 29(1),125-129.

Menna-Barreto, L., Soussignan, R., Montagner, H., \& Koch, P. (1989). The sleep-wake cycle in 4 to 14-months old children: General aspects and sex differences. Brazilian Journal of Medical and Biological Research, 22, 103-106.

Monk, T. H. (1989). Circadian rhythm. Clinical Geriatric Medicine, 5, 331-346.

Monk, T. H., Buysse, D. J., Reynolds Iii, C. F., Kupfer, D. J., \& Houck, P. R. (1995). Circadian temperature rhythms of older people. Experimental Gerontology, 30(5), 455-474.

Moore-Ede, M. C., Sulzman, F. M., \& Fuller, C. A. (1982). The clock that time us. Cambridge: Harvard University Press.

Moore, R. Y., \& Lenn, N. J. (1972). A retinohypothalamic projection in the rat. The Journal of Comparative Neurology, 146, 114.

Moreno, C. R. C., Fischer, F. M., \& Rotenberg, L. (2002). Tolerancia al trabajo en turnos y noturnos: una cuestión multidimensional. In D. Golombek (Org.), Cronobiología humana: Ritmos y relojes biológicos en la salud y en la enfermedad. Buenos Aires: Universidad Nacional de Quilmes Ediciones.

Pandi-Perumal, S. R., Seils, L. K., Kayumov, L., Ralph, M. R., Lowe, A., Moller, H., et al. (2002). Senescence, sleep, and circadian rhythms. Ageing Research Reviews, 1, 559604.

Parmelee, A. H. (1961). Sleep patterns of the newborn. The Journal of Pediatrics, 58(2), 241-250.

Renfrew, J. W., Pettigrew, K. D., \& Rapoport, S. I. (1987). Motor activity and sleep duration as a function of age in healthy men. Physiology and Behavior, 41, 627-634.

Reppert, S. M. (1992). Pre-natal development of a hypothalamic biological clock. Progress in Brain Research, 93, 119-132. 
Ontogênese do Sistema de Temporização - A Construção e as Reformas dos Ritmos...

Rosenberg, R. S. (1982). Aging and biological rhythms: Complaints of insomnia in the elderly. In E. Haus \& H. S. Kabat (Eds.), Chronobiology. New York: Karger.

Sadeh, A. (2001). Sleeping like a baby: A sensitive and a sensible approach to solving your child's sleep problems. New Haven, CT: Yale University Press.

Sadeh, A., Raviv, A., \& Gruber, R. (2000). Sleep patterns and sleep disruptions in school-age children. Developmental Psychology, 36(3), 291-301.

Samis, H. V., Jr. (1968). Aging: The lost of temporal organization. Perspectives in Biology and Medicine, 12(1), 95-102.

Seong, K. H., Ogashiwa, T., Matsuo, T., Fuyama, Y. \& Aigaki, T. (2001). Application of the gene search system to screen for longevity genes in Drosophila. Biogerontology, 2, 209217.

Siegmund, R., Tittel, M., \& Schiefenhövel, W. (1995). Parent-child interaction during activity and rest behavior of inhabitants of Trobriand Islands (Papua New Guinea). Wien Med Wochenschr, 145, 464-467.

Stampi, C. (Org). (1992). Why we nap: Evolution, chronobiology, and functions of polyphasic and ultrashort sleep. Boston: Birkhäuser.

Turek, F. W., Penev, P., Plamen, P., Zhang, Y., Van Reeth, O. \& Zee, P. (1995). Effects of age on the circadian system. Neuroscience and Biobehavioral Reviews, 19(1), 53-58.

Valdez, P., Ramirez, C., \& Garcia, A. (1996). Delaying and extending sleep during weekend: Sleep recovery of circadian effect? Chronobiology International, 13(3), 191-198.

Van Gool W. A., \& Mirmiran M.(1986). Aging and circadian rhythms. Progress in Brain Research, 70, 255-277.

Webb, W. B. (1987). Sleep, biological rhythms and aging. In H. V. Samis \& S. Capobianco (Eds.). Aging and biological rhythms (pp. 309-323). New York: Plenum.

Wehr, T. A., \& Wirz-Justice, A. (1982). Circadian mechanisms in affective illness and in antidepressant drug action. Pharmacopsychiatry, 15, 317-326.

Weissbluth, M. (1995). Naps in children: 6 months - 7 years. Sleep, 19(2), 82-87.

Wey, D (2002). Ciclo vigília/sono de crianças: transição da Educação Infantil para o Ensino Fundamental. Dissertação de Mestrado. Instituto de Psicologia, Universidade de São Paulo, São Paulo.

Recebido em: 3.03.2005

Aceito em: 7.05.2005 
